\title{
Optimal Feedback Controlled Assembly of Perfect Crystals
}

\author{
Xun Tang, $*$ Bradley Rupp, $+\neq$ Yuguang Yang, $\neq \neq$ Tara D. Edwards, $\dagger$ Martha A. Grover, $* \xi$
}

Michael A. Bevants

\section{Supplementary Movies}

Supplementary Movie S1. The first 10 cycles of 200 total uncontrolled and controlled cycles (plotted in Fig. 4a). Even cycles use the control policy, and odd cycles only use the highest $\lambda$. Cycles 2, 6, and 8 use control to achieve perfect crystals, whereas cycles $1,3,4,7,9$, and 10 achieve perfect crystals on their first compression step. Cycle 5 is the only cycle of the first ten to not form a perfect crystal. It forms a near perfect bicrystal that does not relax for $1000 \mathrm{~s}$.

Supplementary Movie S2. Animation of a representative controlled cycle (59th controlled cycle) showing: (left) free energy landscapes and trajectory, (middle) control policy and trajectory, and (right) unprocessed video. Control policy updates shown at $100 \mathrm{~s}$ intervals.

\section{Supplementary Text}

Brownian Dynamics Simulations: Brownian Dynamics (BD) simulations in the canonical ensemble were performed for 300 colloidal particles using numerical methods described in our previous papers. ${ }^{1-5}$ A $0.1 \mathrm{~ms}$ time step was used for at least $2 \times 10^{7}$ steps, and reaction coordinates were stored every 1250 steps for subsequent analysis. Particles in simulations were confined within 2D planes. In the following sections, we provide additional details of the BD simulations, as well

\footnotetext{
* Chemical \& Biomolecular Engineering, Georgia Institute of Technology, Atlanta, GA

$\dagger$ Chemical \& Biomolecular Engineering, Johns Hopkins University, Baltimore, MD

$\$$ These authors contributed equally to this work.

$\S$ email: mabevan@jhu.edu, martha.grover@chbe.gatech.edu
} 
as the models for the conservative forces based on potentials measured in our previous work. ${ }^{6-8}$ All parameters used in the BD simulations are reported in Supplementary Table 1. The BD simulations were based on a Langevin equation as,

$$
m \frac{d \mathbf{U}}{d t}=\mathbf{F}^{H}+\mathbf{F}^{P}+\mathbf{F}^{B}
$$

where $m$ is the particle buoyant mass, $\mathbf{U}$ is a velocity vector, and the force vector has three parts including dissipative hydrodynamic forces, $\mathbf{F}^{H}$, conservative forces due to potential fields, $\mathbf{F}^{P}$, and

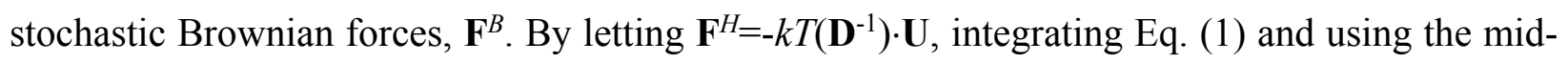
point algorithm, an equation of motion for particle displacements is obtained as, ${ }^{9-10}$

$$
\mathbf{r}=\mathbf{r}^{0}+\left(\nabla \cdot \mathbf{D}^{0}\right) \Delta t+(k T)^{-1} \mathbf{D}^{0} \cdot\left(\mathbf{F}^{P, 0}+\mathbf{F}^{B, 0}\right) \Delta t
$$

where the superscript " 0 " indicates quantities computed at the beginning of the time interval. Specific details of implementing Eq. (2) in dynamic simulations are described in previous publications. ${ }^{1-2}$ The Brownian force $\mathbf{F}^{B}$ is characterized by a mean and variance given by,

$$
\begin{gathered}
\left\langle\mathbf{F}^{B}\right\rangle=\mathbf{0} \\
\left\langle\mathbf{F}^{B}(0) \mathbf{F}^{B}(t)\right\rangle=2(k T)^{2}\left(\mathbf{D}^{-1}\right) \delta(t)
\end{gathered}
$$

D in Eqs. (2) and (3) is a diffusivity tensor for finite numbers of particles above a no-slip plane computed using the methods of Brady and co-workers. D is related to the resistance tensor, $\mathbf{R}$, through the generalized Stokes-Einstein relation, $\mathbf{D}=k T \mathbf{R}^{-1}$. Here $\mathbf{R}$ is computed to include hydrodynamic interactions, which are separated into far-field, multi-body, and near-field lubrication contributions as, ${ }^{11}$

$$
\mathbf{R}=\left(\mathbf{M}^{\infty}\right)^{-1}+\mathbf{R}_{2 B}-\mathbf{R}_{2 B}^{\infty}
$$


where $\mathbf{M}^{\infty}$ is the far-field mobility tensor constructed in a pairwise manner. The inverse of $\mathbf{M}^{\infty}$ is a true multi-body, far-field approximation to the resistance tensor. Lubrication is included by adding the exact two-body resistance tensor, ${ }^{12-14} \mathbf{R}_{2 B}$, and subtracting the two-body, far-field resistance tensor, $\mathbf{R}_{2 B}{ }^{\infty}$, to avoid double counting. To specify an approximate configurationdependent $\mathbf{D}$ to reduce computational cost, the diagonal elements of $\mathbf{D}$ (without cross terms) are parameterized in a look up table as a function of the ensemble $R_{\mathrm{g}}$ and the distance, $R_{\mathrm{i}}$, of particle $i$ from the configuration center of mass.

The interaction energy between colloids within the quadrupole electrode is modeled as the superposition of electrostatic double layer repulsion, dipole-field interactions, and dipole-dipole interactions. The net conservative forces, $\mathbf{F}^{P}$, in Eq. (2) are calculated based on the total conservative force acting on particle $i$ as,

$$
\mathbf{F}_{i}^{P}=-\nabla_{r_{i}}\left[u_{d e, i}^{p f}+\sum_{j \neq i}\left(u_{e, i, j}^{p p}+u_{d d, i, j}^{p p}\right)\right]
$$

where the electrostatic potential between particles $i$ and $j, u^{\mathrm{pp}} \mathrm{e}, \mathrm{i},(\mathbf{r})$, is given by, ${ }^{15}$

$$
u_{e, i, j}^{p p}\left(r_{i j}\right)=32 \pi \varepsilon_{m} a\left(\frac{k T}{e}\right)^{2} \tanh ^{2}\left(\frac{e \psi}{4 k T}\right) \exp \left[-\kappa\left(r_{i j}-2 a\right)\right]
$$

where $r_{i j}$ is the particle center-to-center distance, $e$ is the elemental charge, and $\psi$ is the colloid surface potential. Dipole-field interactions can be described by, ${ }^{6}$

$$
u_{d e, i}^{p f}\left(\mathbf{r}_{i}\right)=-2 k T \lambda f_{c m}^{-1}\left(\frac{E\left(\mathbf{r}_{i}\right)}{E_{0}}\right)^{2}
$$

where $\mathbf{r}_{i}$ is the position of particle $i$ relative to the center of the quadrupole electrode and $E\left(\mathbf{r}_{i}\right)$ is the electric field. Dipolar interactions between particles $i$ and $j$ are given by, ${ }^{7}$ 


$$
u_{d d, i, j}^{p p}(\mathbf{r})=-k T \lambda P_{2}\left(\cos \theta_{i j}\right)\left(\frac{2 a}{r_{i j}}\right)^{3}\left(\frac{E\left(\mathbf{r}_{i}\right)}{E_{0}}\right)^{2}
$$

where $P_{2}\left(\cos \theta_{\mathrm{ij}}\right)$ is the second Legendre polynomial and $\theta_{i j}$ is the angle between the line that connects the two particle centers and the electric field. The quadrupole electric field is given by an approximate expression ${ }^{16}$ corrected to fit numerical COMSOL results as,

$$
\left|\frac{E(L)}{E_{0}}\right|=\frac{4 L}{d_{g}}\left[\begin{array}{l}
2.081 \times 10^{-7} L^{4}-1.539 \times 10^{-9} L^{3}+ \\
8.341 \times 10^{-5} L^{2}+1.961 \times 10^{-5} L+1.028
\end{array}\right]
$$

where $x$ and $y$ are Cartesian coordinates with origin at the quadrupole center, and $L=\left(x^{2}+y^{2}\right)^{0.5}$.

Equilibrated $\psi_{6}$ values: Equilibrium $\psi_{6}$ values in Fig. 2 were obtained from BD simulations using the following procedure: (1) simulations at each $\lambda$ were initiated from fluid-like configurations to construct free energy landscapes by fitting the Smoluchowski equation coefficients (described below), (2) equilibrated $\psi_{6}$ values at each $\lambda$ were obtained as the global free energy minimum on each landscape.

Smoluchowski equation coefficients: Methods to fit the Smoluchowski equation coefficients are described in detail in our previous manuscripts. ${ }^{4-5,17}$ Here we describe in brief the linear fitting method. The local drift and diffusion coefficients are obtained using, ${ }^{18}$

$$
\begin{gathered}
\mathbf{D}_{i}^{(1)}(\mathbf{x})=\left.\lim _{\tau \rightarrow 0} \frac{1}{\tau}\left\langle\chi_{i}(t+\tau)-\chi_{i}(t)\right\rangle\right|_{\chi(t)=\mathbf{x}}=\frac{\partial \mu_{i}}{\partial t} \\
\mathbf{D}_{i j}^{(2)}(\mathbf{x})=\left.\lim _{\tau \rightarrow 0} \frac{1}{2 \tau}\left\langle\left[\chi_{i}(t+\tau)-\chi_{i}(t)\right]\left[\chi_{j}(t+\tau)-\chi_{j}(t)\right]\right\rangle\right|_{\chi(t)=\mathbf{x}}=\frac{1}{2} \frac{\partial \sigma_{i j}^{2}}{\partial t}
\end{gathered}
$$

where $\mathbf{D}^{(1)}$ is the drift vector field, $\mathbf{D}^{(2)}$ is the diffusivity tensor, $\chi$ is a particular realization of $\mathbf{x}$, the brackets represent an ensemble average, and the right hand side provides compact notation 
based on the definition of a derivative and traditional symbols of statistics (i.e., mean, variance, covariance). The free energy landscape, $W(\mathbf{x})$, is obtained from $\mathbf{D}^{(1)}$ and $\mathbf{D}^{(2)}$ as,

$$
\frac{W\left(\mathbf{x}_{2}\right)-W\left(\mathbf{x}_{1}\right)}{k T}=-\int_{\mathbf{x}_{1}}^{\mathbf{x}_{2}}\left(\mathbf{D}^{(2)}\right)^{-1} \cdot\left(\mathbf{D}^{(1)}-\nabla \cdot \mathbf{D}^{(2)}\right)
$$

Here, $\mathbf{x}=\left(\psi_{6}, \mathrm{C}_{6}\right)$ trajectories for input into Eqs. (10) and (11) were generated from $>3000$ simulations initiated from a library of experimental fluid and polycrystalline configurations. Trajectories were analyzed on $\left(\psi 6, \mathrm{C}_{6}\right)$ grid points with at least 4200 trajectories passing through each grid point. The resolution for the $\mathrm{C}_{6}$ and $\psi_{6}$ coordinates were 0.025 and 0.025 .

\section{Supplementary Table}

Supplementary Table 1. Parameters for experiments and simulations. a. particle diameter, ${ }^{19}$

b. Debye screening length, c. particle and wall Stern potential, ${ }^{19}$ d. applied peak-to-peak voltage, e. dimensionless electric field strengths used for policy control, f. Clausius-Mossotti factor for an AC field frequency at $1 \mathrm{MHz},{ }^{7}$ g. medium dielectric permittivity, h. electrode spacing. ${ }^{20}$

\begin{tabular}{|c|c|c|}
\hline Variable & Theory/Simulation & Experiment \\
\hline $2 a / \mathrm{nm}^{\mathrm{a}}$ & 2870 & 2870 \\
\hline$T / \mathrm{K}$ & 293 & 293 \\
\hline$\kappa^{-1} / \mathrm{nm}^{\mathrm{b}}$ & 10 & 10 \\
\hline$\psi / \mathrm{mV}^{\mathrm{c}}$ & -50.0 & -50.0 \\
\hline$V_{\mathrm{pp}} / \mathrm{V}^{\mathrm{d}}$ & $0.2,0.4,0.6,1.9$ & $0.2,0.4,0.6,1.9$ \\
\hline$\lambda^{\mathrm{e}}$ & $0.2,0.9,2.0,19.7$ & $0.2,0.9,2.0,19.7$ \\
\hline$f_{\mathrm{CM}}^{\mathrm{f}}$ & -0.4667 & -0.4667 \\
\hline$\varepsilon_{\mathrm{m}}$ & 78 & 78 \\
\hline$d_{\mathrm{g}} / \mu^{\mathrm{g}}$ & 96 & 96 \\
\hline
\end{tabular}

\section{Supplementary References}

1. Anekal, S.; Bevan, M. A., Interpretation of Conservative Forces from Stokesian Dynamic Simulations of Interfacial and Confined Colloids. J. Chem. Phys. 2005, 122, 034903.

2. Anekal, S.; Bevan, M. A., Self Diffusion in Sub-Monolayer Colloidal Fluids Near a Wall. $J$. Chem. Phys. 2006, 125, 034906. 
3. Anekal, S. G.; Bahukudumbi, P.; Bevan, M. A., Dynamic Signature for the Equilibrium Percolation Threshold of Attractive Colloidal Fluids. Phys. Rev. E 2006, 73, 020403.

4. Beltran-Villegas, D. J.; Sehgal, R. M.; Maroudas, D.; Ford, D. M.; Bevan, M. A., FokkerPlanck Analysis of Separation Dependent Potentials and Diffusion Coefficients in Simulated Microscopy Experiments. J. Chem. Phys. 2010, 132, 044707.

5. Beltran-Villegas, D. J.; Sehgal, R. M.; Maroudas, D.; Ford, D. M.; Bevan, M. A., A Smoluchowski Model of Crystallization Dynamics of Small Colloidal Clusters. J. Chem. Phys. 2011, 135, 154506.

6. Juarez, J. J.; Cui, J.-Q.; Liu, B. G.; Bevan, M. A., kT-Scale Colloidal Interactions in High Frequency Inhomogeneous AC Electric Fields. I. Single Particles. Langmuir 2011, 27, 92119218.

7. Juarez, J. J.; Liu, B. G.; Cui, J.-Q.; Bevan, M. A., kT-Scale Colloidal Interactions in HighFrequency Inhomogeneous AC Electric Fields. II. Concentrated Ensembles. Langmuir 2011, 27, 9219-9226.

8. Juarez, J. J.; Bevan, M. A., Interactions and Microstructures in Electric Field Mediated Colloidal Assembly. J. Chem. Phys. 2009, 131, 134704.

9. Ermak, D. L.; McCammon, J. A., Brownian Dynamics with Hydrodynamic Interactions. $J$. Chem. Phys. 1978, 69, 1352-1360.

10. Grassia, P. S.; Hinch, E. J.; Nitsche, L. C., Computer-Simulations of Brownian-Motion of Complex-Systems. J. Fluid Mech. 1995, 282, 373-403.

11. Swan, J. W.; Brady, J. F., Simulation of Hydrodynamically Interacting Particles Near a NoSlip Boundary. Phys. Fluid 2007, 19, 113306.

12. Jeffrey, D. J.; Onishi, Y., Calculation of the Resistance and Mobility Functions for two Unequal Rigid Spheres in Low-Reynolds-Number Flow. J. Fluid Mech. 1984, 139, 261-290.

13. Brenner, H., The Slow Motion of a Sphere Through a Viscous Fluid Towards a Plane Surface. Chem. Eng. Sci. 1961, 16, 242-251.

14. Goldman, A. J.; Cox, R. G.; Brenner, H., Slow Viscous Motion of a Sphere Parallel to a Plane Wall -- I. Motion Through a Quiescent fluid. Chem. Engr. Sci. 1967, 22, 637-651.

15. Russel, W. B.; Saville, D. A.; Schowalter, W. R., Colloidal Dispersions. Cambridge University Press: New York, 1989.

16. Huang, Y.; Pethig, R., Electrode Design for Negative Dielectrophoresis. Meas. Sci. Technol. 1991, 2, 1142-1146.

17. Beltran-Villegas, D. J.; Sehgal, R. M.; Maroudas, D.; Ford, D. M.; Bevan, M. A., Colloidal Cluster Crystallization Dynamics. J. Chem. Phys. 2012, 137, 134901.

18. Risken, H., The Fokker-Planck Equation: Methods of Solution and Applications. Second ed.; Springer: Berlin, 1996; Vol. 18.

19. Wu, H. J.; Bevan, M. A., Direct Measurement of Single and Ensemble Average ParticleSurface Potential Energy Profiles. Langmuir 2005, 21, 1244-1254.

20. Juarez, J. J.; Feicht, S. E.; Bevan, M. A., Electric Field Mediated Assembly of Three Dimensional Equilibrium Colloidal Crystals. Soft Matter 2012, 8, 94-103. 